\title{
6.6 NUCLEAR FORCES IN HIGH DENSITY MATTER
}

\author{
M. R. McNAUGHTON
}

Laboratory for Astrophysics and Space Research, Enrico Fermi Institute, Chicago, Ill., U.S.A.

\begin{abstract}
The conditions for superfluidity or ferromagnetism in neutron stars are presented and discussed (but not derived). It is suggested that present estimates relating to these are in error and that the predictions made contradict at least one of three sets of nuclear physics data cited in the text. This is due to neglecting the action of the exclusion principle.

A comparatively simple method for calculating the strength of nuclear forces in the presence of many-body effects is outlined. Some preliminary results are presented together with projected future developments.
\end{abstract}

\section{Introduction}

Figure 1 shows the composition of neutron star matter at nuclear densities and zero temperature. This is computed assuming the particles obey the exclusion principle, are in chemical equilibrium, and form a medium that has over-all charge neutrality. Otherwise the interparticle forces have been neglected. The most significant feature of the diagram is the behaviour of the proton kinetic energy. This is very low compared to the neutron kinetic energy until $\Sigma^{-}$particles are formed, then it rises to a similar value.

When strong interactions between the particles are introduced, the physical effects can be roughly divided according to whether the interaction between particles of the same type is attractive or repulsive. If the forces are sufficiently attractive then the corresponding particles will form a superfluid; on the other hand, repulsive forces may cause ferromagnetism. The equations regulating these two effects are found to be

$$
\begin{aligned}
& \delta_{k}=-\frac{1}{2} \sum_{\bar{k}^{\prime}} \frac{\delta_{k^{\prime}}\left\langle\bar{k},-k\left|T^{(S)}\right| k^{\prime},-k^{\prime}\right\rangle}{\left[\delta_{\bar{k}^{\prime}}^{2}+\left(E_{k^{\prime}}-E_{k_{\mathbf{F}}}\right)^{2}\right]^{1 / 2}} \\
& 1 \leqslant N(0)\left\{T^{(S)}-\bar{T}^{(t)}\right\} .
\end{aligned}
$$

$\delta_{k}$ is the superfluid energy gap experienced by a particle in momentum state $\bar{k}$ which has an energy $E_{k} . N(0)$ is the density of states per unit energy range close to the Fermi surface. The first equation is the well known first order B.C.S. equation. The matrix element it contains is derived from the scattering of particles with momentum $\left(\bar{k},-\bar{k}^{\prime \prime}\right)$ into states with momentum $(k,-k)$. The second equation is the criterion for saturated ferromagnetism. The matrix elements represent the forces acting between typical particles in the singlet or triplet configurations. The criterion is that the forces be less repulsive if all the particles have the same spin direction, this state will then be energetically favoured and a net magnetic dipole will be associated with the preferred spin direction.

The difficulty in utilizing Equations (1) and (2) results almost entirely from correctly evaluating the matrix elements. Present estimates are usually based on neglecting the 
exclusion principle (superfluidity; Hoffberg et al., 1970), or treating the particles as repulsive spheres (ferromagnetism; Brownell and Collaway, 1969), or taking an oversimplified model interaction (superfluidity; McNaughton, 1969). It is suggested below that all of these approximations are in error.

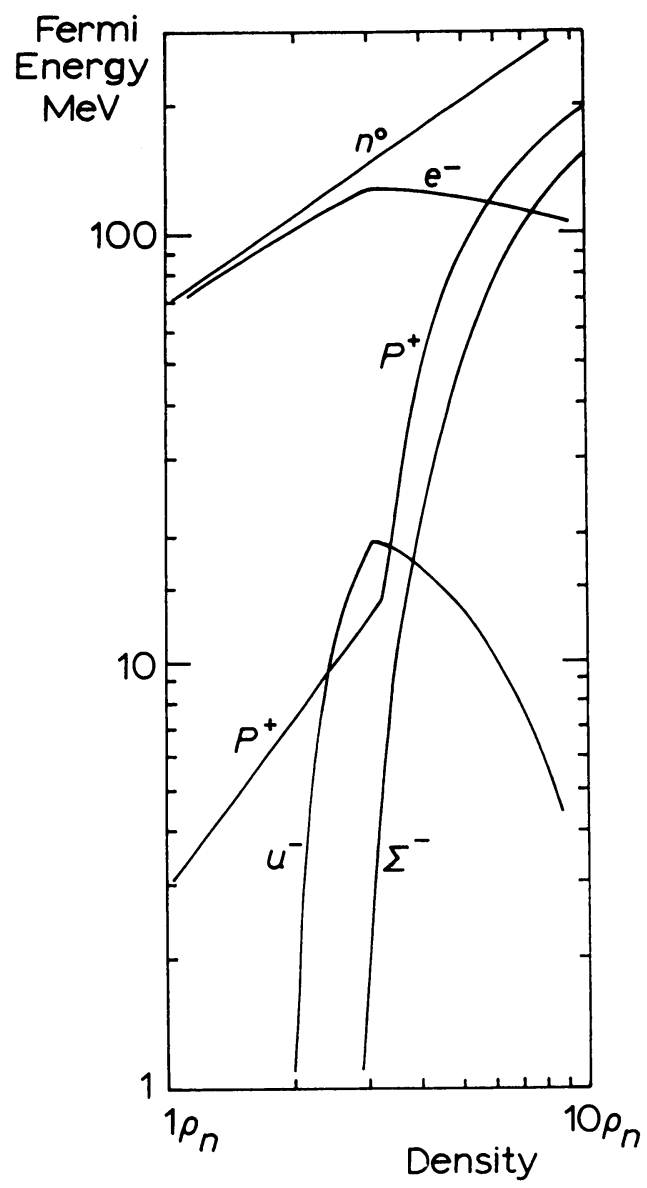

Fig. 1. The Fermi energy of the particles present in neutron star material as a function of the total density. The density is plotted in multiples of the equilibrium density of ordinary nuclear matter, $Q_{n}$, $\left(Q_{n}=3.7 \times 10^{14} \mathrm{~g} \mathrm{~cm}^{-3}\right)$.

Since a theory of nuclear forces cannot be tested directly in the astrophysical context it is advisable to look for laboratory situations to which it can be applied. There are three types of experimental data for which such a theory might reasonably be expected to account. These are:

(i) phase shifts determined from nuclear scattering experiments.

(ii) the nuclear matter binding energy

(iii) even-even nuclei pairing strengths 
Item (i) is a measure of the strength of two body forces without the interference of other particles or the exclusion principle. It may be used to adjust free parameters in an expression for the bare two-nucleon interaction. Item (ii), the nuclear matter binding energy, measures the strength of the attraction seen by typical particles in a fully degenerate nucleon gas at one particular density - the density of ordinary nuclei near their center. Numerically it is about $-16 \mathrm{MeV}$ per particle at the density $\varrho_{n} \equiv 3.7 \times 10^{14} \mathrm{~g} \mathrm{~cm}^{-3}$. The third item is determined from nuclear structure experiments and is basically a measure of the superfluid energy gap found at densities corresponding to the surface layers of nuclei. That the pairing (energy gap) occurs only at the nuclear surface $\left(\varrho \ll \varrho_{n}\right)$ can be seen by noting that the observed pairing energy falls off rapidly with increasing nuclear size (Figure 2). Heavier nuclei have a smaller surface to volume ratio so that low density phenomena are less apparent.

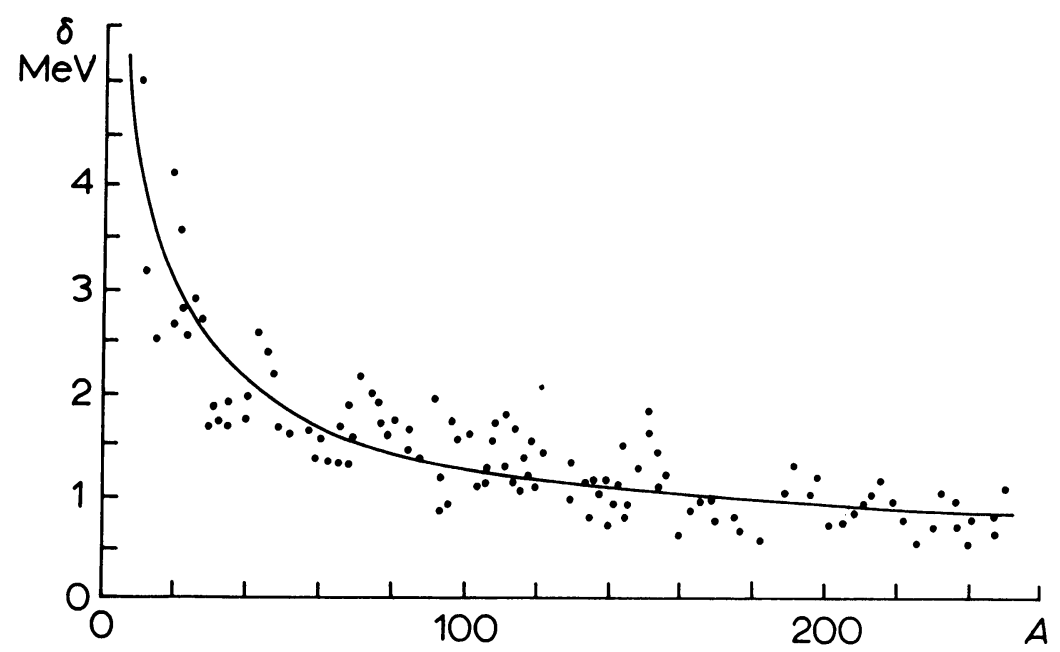

Fig. 2. Pairing strengths measured in nuclei as a function of atomic number, $A$. After averaging over effects caused by partially closed shells, the results fit $\delta=16.2 \times A^{-0.551}$.

\section{Perturbation Diagrams, the $V$ matrix to $T$ matrix transformation}

The simplest nuclear interaction is drawn in Figure 3a. It represents particles of momentum $(k,-k)$ scattering into states $\left(k^{\prime},-\bar{k}^{\prime}\right)$. If the nuclear potential, $V(r)$, contains an infinitely repulsive core, $V(r) \rightarrow \infty$ for $r<r_{c}$, then the corresponding matrix element, $\left\langle\bar{k}^{\prime},-\bar{k}^{\prime}|V| \bar{k},-\bar{k}\right\rangle$, will be infinite. To obtain a finite result, an infinite set of diagrams (Figure 3b, part (2), (3), (4) etc.) must be added together. Qualitatively, this corresponds to allowing for the correlation of the plane wave function about the repulsive core of the other particle. Alternatively, it means summing a perturbation series to infinite order. 
If the sum of this series of diagrams is designated $\left\langle\bar{k}^{\prime},-\bar{k}^{\prime}|T(\omega)| \bar{k},-\bar{k}\right\rangle$ then it obeys the integral equation

$$
\begin{aligned}
& \left\langle\bar{k}^{\prime},-\bar{k}^{\prime}|T(\omega)| \bar{k},-\bar{k}\right\rangle=\left\langle\bar{k}^{\prime},-\bar{k}^{\prime}|V| \bar{k},-\bar{k}\right\rangle \\
& -\sum_{\bar{k}^{\prime \prime}} \frac{u_{\bar{k}^{\prime \prime}}^{2} u_{-\bar{k}^{\prime \prime}}^{2}\left\langle\bar{k}^{\prime}-\bar{k}^{\prime}|V| \bar{k}^{\prime \prime}-\bar{k}^{\prime \prime}\right\rangle\left\langle\bar{k}^{\prime \prime}-\bar{k}^{\prime \prime}|T(\varpi)| \bar{k}-\bar{k}\right\rangle}{\left[E_{\bar{k}^{\prime \prime}}+E_{-\bar{k}^{\prime \prime}}+\omega\right]} \\
& -\varpi=E_{\bar{k}}+E_{-\bar{k}}
\end{aligned}
$$

$u_{\bar{k}^{\prime \prime}}^{2}$ and $E_{\bar{k}^{\prime \prime}}$ measure the availability and total energy of the momentum state $\bar{k}^{\prime \prime}$. In a laboratory scattering situation they are replaced by 1 and $(\hbar k)^{2} / 2 m$ respectively. It is convenient to represent the sum of these interactions by a single wavy line diagram (Figure 3b, part (1)).

(a)

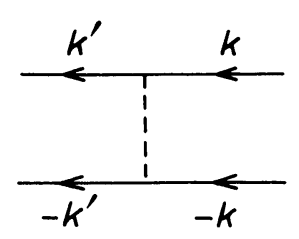

(b)

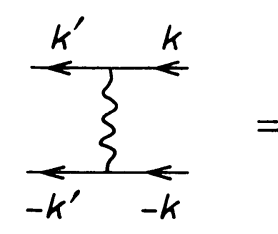

1 (c)

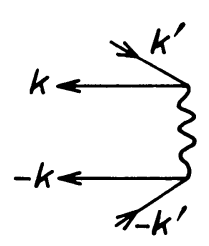

(d)

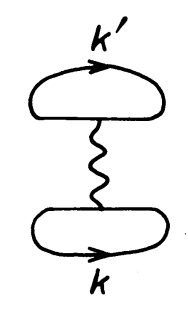

Fig. 3. (a) shows a simple scattering diagram, the corresponding matrix element is $\left\langle-k^{\prime}, k^{\prime}|V|-k, k\right\rangle$. (b) illustrates how the $T$ matrix is defined. (c) and (d) are the diagrams that define the matrix elements in equations (1) and (2) respectively.

The matrix elements occurring in Equations (1) and (2) obey very similar equations. The corresponding diagrams are drawn in Figures $3 \mathrm{c}$ and $3 \mathrm{~d}$. The backward pointing lines refer to holes in the Fermi sea and give a negative contribution to the energy denominators in the equations corresponding to Equation (3). The present work is directed to solving equations of this type having proper regard to the exclusion principle, $\left(u_{k^{\prime \prime}}^{2}=0\right.$ for $\left.k^{\prime \prime}<k_{\mathrm{F}}\right)$, and to the influence of the other particles on the self consistent energies, $\left(E_{k^{\prime \prime}} \neq(\hbar k)^{2} / 2 m\right)$. 
The starting point in the calculation is a separable potential for the bare two body interaction:

$$
V_{k^{\prime} k} \equiv\left\langle\bar{k}^{\prime},-\bar{k}^{\prime}|V| \bar{k},-\bar{k}\right\rangle=\sum_{\alpha} \lambda_{\alpha} W_{\alpha}\left(k^{\prime}\right) W_{\alpha}(k)
$$

The functions $W_{\alpha}(k)$ are chosen to be easy to use and the constants $\lambda_{\alpha}$ are then adjusted so that the phase shifts predicted agree with the experimental values. The solution to Equation (3) can then be written in the form

$$
T_{k^{\prime} k}(\omega) \equiv\left\langle\bar{k}^{\prime},-\bar{k}^{\prime}|T(\omega)| \bar{k},-\bar{k}\right\rangle=\sum_{\alpha \alpha^{\prime}} A_{\alpha \alpha^{\prime}} W_{\alpha}\left(k^{\prime}\right) W_{\alpha^{\prime}}(k) .
$$

The matrix $A$ is found to obey

with

$$
A^{-1}=(D+M)
$$

$$
\begin{aligned}
D_{\alpha \alpha^{\prime}} & =\lambda_{\alpha}^{-1} \delta_{\alpha \alpha^{\prime}} \\
M_{\alpha \alpha^{\prime}} & =\sum_{\bar{k}^{\prime \prime}} \frac{u_{k^{\prime \prime}}^{2} u_{-k^{\prime \prime}}^{2}}{E_{k^{\prime \prime}}+E_{-k^{\prime \prime}}+\omega} W_{\alpha}\left(k^{\prime \prime}\right) W_{\alpha^{\prime}}\left(k^{\prime \prime}\right) .
\end{aligned}
$$

To evaluate the integrals in (6.3) we borrow an idea from the reference spectrum method of nuclear theory (Day, 1967) and put

$$
\begin{array}{ll}
E_{k}=(\hbar k)^{2} / 2 m_{1}^{*}+u_{1}, & k<k_{\mathrm{F}} \\
E_{k}=(\hbar k)^{2} / 2 m_{2}^{*}+u_{2}, & k>k_{\mathrm{F}}
\end{array}
$$

where $u$ and $m^{*}$ are constants.

Only one form of $V_{k^{\prime} k}$ has been investigated to date, this is a Puff type potential for the singlet $S$ state:

$$
\begin{aligned}
& \qquad V_{k^{\prime} k}^{(S)}=2(2 \pi)^{3} \underset{\lambda_{c} \rightarrow \infty}{\operatorname{Lt}}\left[\lambda_{c} \frac{\sin \left(k^{\prime} r_{c}\right)}{k^{\prime}} \frac{\sin \left(k r_{c}\right)}{k^{\prime}}-\frac{\lambda}{\left(k^{\prime 2}+\beta^{2}\right)\left(k^{2}+\beta^{2}\right)}\right] \\
& \hbar=2 m=1 \\
& \lambda=0.886 \mathrm{~F}^{-3} \\
& \beta=1.62 \mathrm{~F}^{-1} \\
& r_{c}=0.257 \mathrm{~F}
\end{aligned}
$$

The details of matching this to the experimental phase shifts are not reproduced here but it is important to obtain a good fit, not only at energies corresponding to $k$ and $k^{\prime}(\simeq 10 \mathrm{MeV})$, but also at much higher energies $(\simeq 400 \mathrm{MeV})$ if the integral in equation (6.3) is to be correct. Using potentials of this form the reaction matrix, $T_{k^{\prime} k}$, can be found analytically. The constants $u$ and $m^{*}$ may be determined self consistently by computing the value of the diagrams that give rise to the particle binding energies. Because of the integrals involved in (6.3), the expressions are lengthy and are best displayed in numerical form as graphs. In practice the excited particle states may be treated as free particles $\left(u_{2}=0, m_{2}^{*}=1\right)$. The corresponding quantities for states below the Fermi surface are typically $u_{1} \simeq-100 \mathrm{MeV}$ and $m_{1}^{*} \simeq 0.6$. 


\section{Numerical Results}

Figure 4 shows the behaviour of $T_{k k}^{(S)}$ against $k$ for a neutron-proton gas ('nuclearmatter') at a density $\varrho=\varrho_{n}$. The result obtained by ignoring the exclusion principle and many-body forces,

$$
T_{k k}^{*}=-\left(\frac{4 \pi}{m k}\right) \delta(k) \quad \delta(k)=\text { phase shift } .
$$

is also shown. The agreement is very poor.

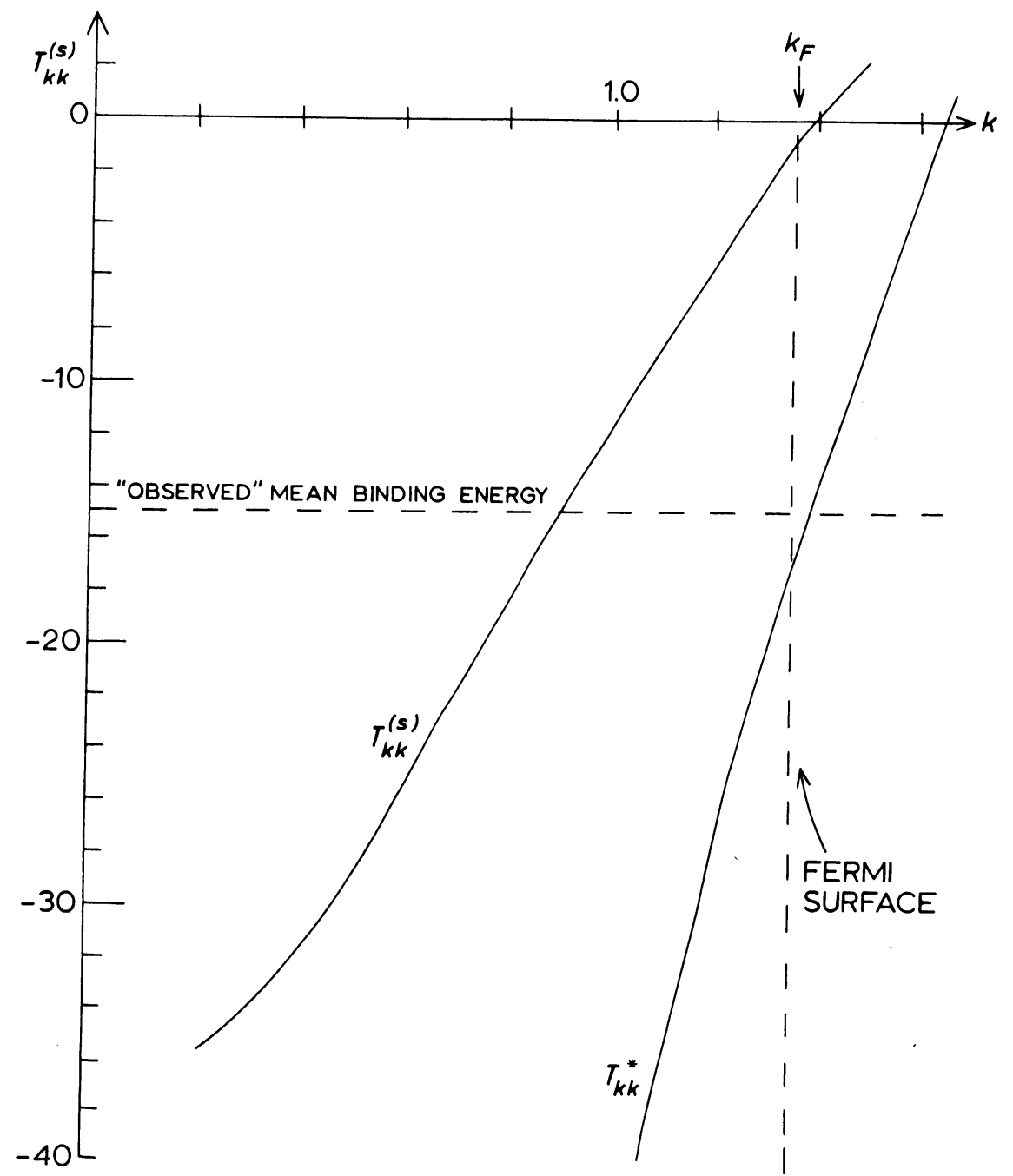

Fig. 4. $T_{k k}$ as a function of momentum, $k$. The units are derived with $2 m=h=1 ; T_{k k}$ is in F and $k$ is in $\mathrm{F}^{-1}\left(1 \mathrm{~F}=10^{-13} \mathrm{~cm}\right) . T_{k k^{*}}$ gives a poor approximation to the observed mean binding energy, it also predicts a large negative value of the matrix element at the Fermi surface. 
The scattering approximation gives much more negative values for $T_{k k}^{(S)}$ and, as a result, a very large net binding energy $(\simeq 130 \mathrm{MeV})$. This may be understood by inspecting the phase shift against momentum diagram, Figure 5. The lowest momentum states see the most attractive potential, but these are just the states that are excluded from the summation over intermediate states by the exclusion principle.

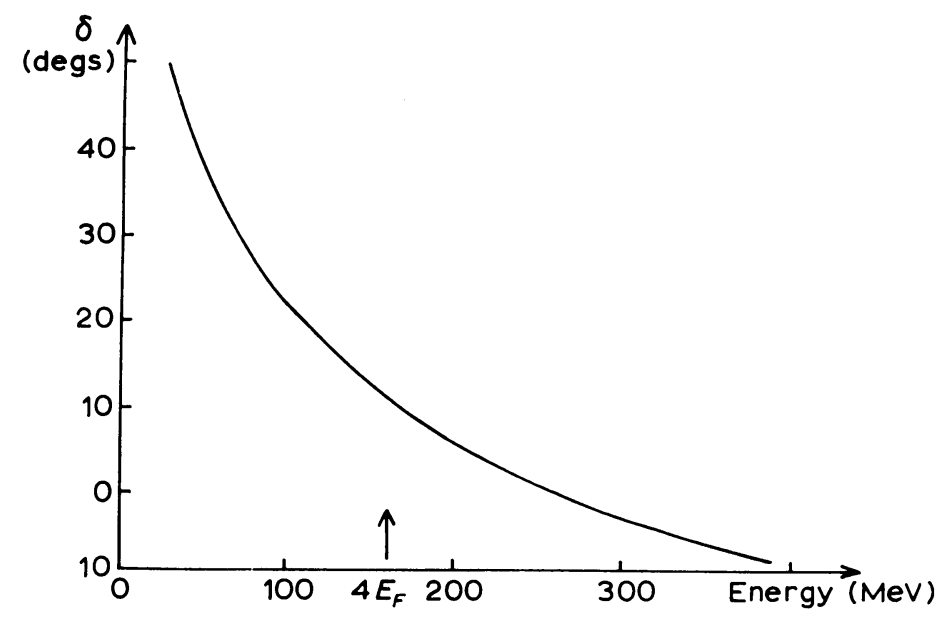

Fig. 5. Phase shift, $\delta$, observed in the ${ }^{1} S_{0}$ configuration plotted against the incident particle energy. The point marked $4 E_{\mathrm{F}}$ corresponds to the Fermi energy used in Figure 4.

The size of the superfluid energy gap calculated from Equation (1) depends on the value of $T_{k k}^{(S)}$ near the Fermi surface. For a finite gap, we require,

$$
N(0) T_{k_{\mathrm{F}} k_{\mathrm{F}}}^{(S)} \approx-1 \text {. }
$$

The energy gap predicted by including the exclusion principle is vanishingly small. Equation (8), on the other hand would predict a large effect at nuclear density and consequently an even-even nuclei pairing energy of several $\mathrm{MeV}$ independent of the nuclear size.

\section{Conclusions, Future Development}

We believe that the techniques of Section 2 give results demonstrably in agreement with the three experimental tests referred to in Section 1. The step 'backwards' from the well known phase shifts before re-calculating the $T$ matrix is an unfortunate necessity if useful results are to be obtained.

Further work is expected to show that the critical Fermi energy above which equation (9) fails is in the region $30<E_{\mathrm{F}}<40 \mathrm{MeV}$. If this is the case, the shape of the proton Fermi energy curve in Figure 1 is indeed fortunate, since it will single out the protons at $\varrho<2 \times 10^{15} \mathrm{~g} \mathrm{~cm}^{-3}$ for an energy gap of several $\mathrm{MeV}$. The exact details of the $\lambda=\lambda\left(E_{\mathrm{F}}\right)$ relation will have no observationally significant results. That the neutrons may satisfy the Inequality (9) at sufficiently low densities is not important 
since a neutron star with an average density less than $\varrho_{n}$ is unlikely to be stable (Wang et al., 1970).

Hoff berg et al. have suggested that superfluidity may result from the coupling of particles with their spins parallel. In this case the interaction proceeds via the $P$ state potential which remains attractive at higher values of $E_{\mathrm{F}}$ after the $S$ state interaction has turned repulsive. While the $S$ state interaction cannot enter into the first order matrix element in Equation (1), it may enter through higher order diagrams (Figure 6).

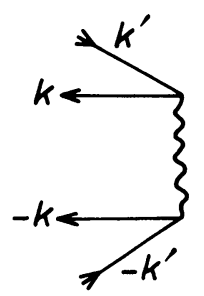

(a)

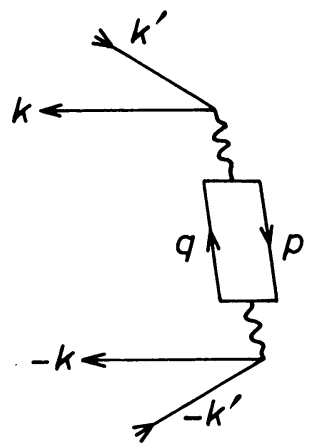

(b)

Fig. 6. (a) shows the first order matrix element of the B.C.S. equation. (b) shows the second order matrix elements that enter into higher order approximations.

Present indications are that the large repulsive contribution of the higher order diagram may overwhelm the intrinsically smaller attractive contribution of the first order diagram. However, more work is needed on this point.

The parameters of the two-body triplet interaction $V_{k^{\prime} k}^{(T)}$ have been found but the inequality for ferromagnetism has not yet been tested.

\section{References}

Brownell, D. H. and Callaway, J. A.: 1969, Nuovo Cimento 60B, 169.

Day, B. D.: 1967, Rev. Mod. Phys. 39, 719.

Hoffberg, M., Glassgold, A. E., Richardson, R. W., and Ruderman, M.: 1970, Phys. Rev. Letters $24,775$. McNaughton, M. R.: 1969, Thesis, Manchester University.

Wang, C. G., Rose, W. K., and Schlenker, S. L.: 1970, Astrophys. J. 160, L17.

\section{Discussion}

V. Canuto: Are phase shifts a good method for determining the potential to be used? .

M. McNaughton: They do not yield a unique solution for the potential but any potential used must satisfy these results. The point of citing nuclear matter binding energy etc., is to further distinguish between the potentials and select the correct one.

$V$. Canuto: Is the result determined for $S$ waves only?

$M$. McNaughton: Yes. To consider the $P$ and higher states suggested by Hoff berg et al., one must go beyond the simple B.C.S. equation. It is possible for 3 rd order diagrams to give a large (and repulsive) contribution since the 3rd particle is not confined to any special configuration with the two main particles, i.e. the repulsive $S$ state interaction can enter and overcome the $P$ state interaction. 